\title{
Qualidade na Interação Familiar e Estresse Parental e suas Relações com o Autoconceito, Habilidades Sociais e Problemas de Comportamento dos Filhos
}

\author{
Gisele Regina Stasiak \\ Lidia Natalia Dobrianskyj Weber \\ Claudia Tucunduva \\ Universidade Federal do Paraná \\ Curitiba, PR, Brasil
}

\begin{abstract}
RESUMO
Desde o século passado, diversas pesquisas acadêmicas sobre educação de filhos e suas relações com o desenvolvimento psicológico infantil são realizadas. O objetivo da presente pesquisa foi analisar a qualidade na interação familiar e o estresse parental de pais e mães de crianças que estão na fase de transição para o primeiro ano do ensino fundamental e suas relações com autoconceito, habilidades sociais e problemas de comportamento dessas crianças. Os participantes foram 39 crianças $(5,9$ anos), suas mães $(n=39)$ e seus pais $(n=25)$ que responderam as Escalas de Qualidade na Interação Familiar, Inventário de Estresse Parental, Escala de Comportamento Social para Pré-Escolares e Escala de Percepção do Autoconceito Infantil. Os resultados apontaram para várias correlações/relações significativas e estabeleceram três modelos de regressão linear múltipla, tendo a variável habilidades sociais das crianças um valor preditivo em destaque. Portanto, a qualidade na interação familiar pode ser tanto uma fonte decisiva de segurança e bem estar, quanto um ambiente que propicia comportamentos externalizantes e dificulta as construções de um autoconceito positivo e repertório adequado de habilidades sociais de crianças.
\end{abstract}

Palavras-chave: Interação familiar; Estresse parental; Problemas de comportamento.

\begin{abstract}
Quality in Family Interaction and Parental Stress and its Relationships with Children's Self-Concept, Social Skills and Behavior Problems

Diverse academic research about child education and its relationships with children's psychological development has been conducted ever since the last century. The object of this study was to analyze quality in family interaction and parental stress of fathers and mothers whose children who are in the phase of transition to the first year of primary education and the relationships of these factors with these children's self-concept, social skills and behavior problems. The participants were 39 children (5.9 years), their mothers $(n=39)$ and their fathers $(n=25)$ who answered the Family Interaction Quality Scales, the Parental Stress Index, the Preschool and Kindergarten Behavior Skills and the Child Self-Concept Perception Scales. The results indicated several significant correlations/relationships and lead to three models of multiple linear regression, whereby the predictive value of the variable relating to the children's social skills stood out. Quality in family interaction can therefore be both a decisive source of security and well-being and also an environment propitious to externalizing behaviors which hinders children's formation of positive self-concept and an adequate repertoire of social skills.
\end{abstract}

Keywords: Family interaction; Parental stress; Behavior problems.

\section{RESUMEN}

Calidad en la Interacción Familiar y el Estrés Parental y sus Relaciones con el Autoconcepto, Habilidades Sociales y Problemas de Comportamiento de los Hijos

Desde el siglo pasado, diversas investigaciones académicas sobre educación de hijos y sus relaciones con el desarrollo sicológico infantil se lleva a cabo. El objetivo de la presente investigación ha sido lo de analizar la calidad de las interacciones familiares y el estrés parental, de padres y madres de niños que están en la fase de transición para el primer año de la escuela primaria y su relación con el autoconcepto, habilidades sociales y problemas de conducta de esos niños. Los participantes han sido de 39 niños $(5,9$ años), sus madres $(n=39)$ y sus padres $(n=25)$ que han contestado a la Escala de Calidad en la Interacción Familiar, Inventario de Estrés Parental, Escala de Comportamiento Social para Preescolar y Escala de Percepción del Autoconcepto Infantil. Los resultados han apuntado para varias correlaciones/relaciones significativas y han establecido tres patrones de regresión lineal múltiple, y las habilidades sociales de los niños el valor predictivo variable resaltada. Por lo tanto, la calidad de las interacciones familiares puede ser una fuente decisiva de la seguridad y el bienestar, como un ambiente que promueva um comportamiento llamativo y dificulta la construcción de un autoconcepto positivo y las habilidades sociales adecuadas de los niños.

Palabras clave: interacción familiar, estrés parental, problemas de conducta. 
Os estudos atuais acerca da interação familiar concentram-se em pesquisar como são as famílias de crianças que apresentam um desenvolvimento socioemocional pleno e como são as que trazem prejuízos ao desenvolvimento de crianças e adolescentes (Weber, 2008). A importância desses estudos refere-se à concepção de que a família é um dos primeiros ambientes de socialização dos indivíduos (Dessen \& Polonia, 2007; Kreppner, 2000; Weber, 2008) e de que a família, na condição de sistema social, é responsável pela transmissão de valores, crenças e significados presentes nas sociedades (Kreppner, 2000). O ambiente familiar proporciona à criança diferentes aprendizagens, tais como, administrar e resolver conflitos, controlar emoções, expressar sentimentos nas relações interpessoais e lidar com a diversidade e as adversidades da vida (Wagner, Ribeiro, Arteche, \& Bornholdt, 1999).

Buscando aprofundar esse tema, família e desenvolvimento infantil, o objetivo da presente pesquisa foi analisar a qualidade na interação familiar e o estresse parental de pais e mães de crianças que estão na fase de transição para o primeiro ano do ensino fundamental e suas relações com três variáveis: autoconceito, habilidades sociais e problemas de comportamento dessas crianças. A escolha das variáveis é centrada na premissa de que o comportamento humano é multideterminado, ou seja, o processo de socialização sofre influência de diversos fatores, como, por exemplo, as práticas educativas parentais, comportamento da criança, qualidade da relação do casal, número de filhos, escolaridade etc. (Bolsoni-Silva \& Marturano, 2010; Carmo \& Alvarenga, 2012; Freitas \& Piccinini, 2010; Lengua \& Kovacs, 2005; Sampaio \& Vieira, 2010). Dessa forma, pode-se contribuir de maneira preventiva e com grande aplicabilidade em intervenções sociais que envolvam família, crianças e escola.

Em relação à qualidade na interação familiar, uma das funções da família mais pesquisada é a parental (Weber, 2008). As práticas parentais, ou seja, os comportamentos socializadores dos pais em relação à criança, como disciplina e apoio, variam entre as situações vivenciadas pais-criança (Oliveira et al., 2002; Macarini, Martins, Minetto, \& Vieira, 2010). Dentre os vários tipos de comportamentos dos pais que podem estar funcionalmente relacionados ao comportamento dos filhos, as práticas educativas têm se destacado por constituírem comportamentos dos pais reforçados por modificações produzidas no comportamento dos filhos (Alvarenga \& Piccinini, 2001). Os pais tendem a utilizar essas estratégias com o objetivo de suprimir ou eliminar certos comportamentos da criança considerados inadequados ou indesejáveis, ao passo que as ocorrências dos comportamentos adequados são incentivadas e favorecidas por eles (Alvarenga \& Piccinini, 2001).

As funções parentais podem ser permeadas por sofrimento e angústia, interações disfuncionais que não reforçam os papéis de pai e mãe e por influências das características comportamentais da criança que facilitam ou dificultam essas interações (Abidin, 1995). Neste contexto, as práticas de socialização e a criação dos filhos podem se tornar fonte de estresse parental e, consequentemente, interferir no desenvolvimento infantil (Dessen \& Szelbracikowski, 2004; Mckelvey, Fitzgerald, Schiffman, \& Von Eye, 2002; PereiraSilva \& Dessen, 2006). O estresse parental, segundo Deater-Deckard (1998), define-se como uma reação psicológica aversiva às demandas dos papéis de pai e mãe.

Em relação ao desenvolvimento infantil, a primeira das três variáveis selecionadas nesse estudo que pode sofrer influência das práticas educativas e do estresse parentais foi o autoconceito. $\mathrm{O}$ autoconceito caracteriza-se basicamente pelo seu aspecto descritivo, ou seja, refere-se àquilo que cada indivíduo sabe sobre si por meio da experiência, reflexão e feedback do ambiente social (Loos \& Cassemiro, 2010).

Atitudes de superproteção e controle excessivo pelos pais favorecem um autoconceito empobrecido do filho (Sánchez \& Escribano,1999). Bolsoni-Silva e Marturano (2002) apontaram a comunicação com os pais como um aspecto favorecedor à formação do autoconceito satisfatório dos filhos, que os ajuda a enfrentar as situações cotidianas, ou como uma prática educativa parental prejudicial, que influencia o desenvolvimento de problemas de comportamento de crianças (Weber, Viezzer \& Brandenburg, 2004). Rocha, Ingberman e Breus (2011) investigaram o autoconceito em crianças de cinco e seis anos e encontraram que as práticas parentais positivas (incentivo, instrução direta e clara, contato físico não aversivo) parecem contribuir para um autoconceito positivo dos filhos.

A segunda variável investigada no presente estudo foram as habilidades sociais (HS) dos filhos. O termo HS refere-se "à existência de diferentes classes de comportamentos sociais no repertório do indivíduo para lidar de maneira adequada com as demandas das situações interpessoais" (Del Prette \& Del Prette, 2002, p. 31). A influência das práticas educativas parentais na construção do repertório de habilidades sociais dos filhos (Del Prette \& Del Prette, 2006; 2008; Bolsoni-Silva \& Loureiro, 2011) se dá por meio de três alternativas utilizadas pelos pais: "(a) estabelecimento de regras, por meio de orientações, instruções e exortações, (b) manejo de consequências, por meio de 
recompensas e punições, (c) oferecimento de modelos" (Del Prette \& Del Prette, 2006, p. 59).

Segundo Cia, Pamplin e Del Prette (2006), mães com comunicação assertiva e que participam da vida de seus filhos, possuem filhos mais habilidosos socialmente. Del Prette e Del Prette (2006; 2008) enfatizam a importância do feedback positivo fornecido pelos pais aos filhos, pois as consequências positivas incentivam e fortalecem desempenhos que, posteriormente, serão mantidos por consequências naturais. Barros e Del Prette (2007) mostram que pais com alto envolvimento com seus filhos servem como modelo adequado às habilidades sociais dos mesmos. Marin, Piccinini e Tudge (2011) investigaram mães e pais e descobriram que as mães tendem a conversar mais com seus filhos bem como expressar sentimentos, estabelecer limites e elogiar comportamentos adequados.

Além de pesquisas sobre autoconceito e habilidades sociais dos filhos, publicações apontam os problemas de comportamento relacionados às práticas educativas parentais (Bolsoni-Silva, Silveira, \& Marturano, 2008; Weber \& Moura, 2008). Os problemas de comportamento são definidos por Bolsoni-Silva e Del Prette (2003, p. 461) como "excessos ou déficits comportamentais que dificultam o acesso da criança às novas contingências relevantes de aprendizagem, promotoras do desenvolvimento infantil". Eles são classificados em dois grandes grupos: os comportamentos externalizantes e os internalizantes. Os comportamentos externalizantes são mais frequentes em transtornos de agressividade física e/ou verbal, desafiador ou opositor, condutas antissociais e comportamentos de risco como uso de substâncias. Os comportamentos internalizantes são mais identificáveis em transtornos como depressão, ansiedade e fobia social, todos com implicações sobre o isolamento social e o autoconceito (Del Prette \& Del Prette, 2006).

Pesquisas mostram que pais de crianças sem problemas de comportamento apresentam afeto e atitudes positivas (Pettit \& Bates, 1989) e são mais proativos e consistentes em suas práticas educativas parentais (Bolsoni-Silva \& Marturano, 2008; Ferreira \& Marturano, 2002). O estilo autoritário materno e as práticas coercitivas como, por exemplo, as punições corporais mostram-se associados a problemas de comportamento externalizantes e internalizantes dos filhos (Alvarenga \& Piccinini, 2001; Brandenburg \& Weber, 2005; Oliveira \& Caldana, 2009; Oliveira et al., 2002). Já a atitude conjugal conflituosa parece ser preditora de comportamentos externalizantes dos filhos (Oliveira et al., 2002). Comportamento de superproteção, poucas práticas afetuosas e sintomas de ansiedade/depressão dos pais são preditores de problemas de comportamento internalizantes na infância (Bayer, Sanson, \& Hemphill, 2006). Estudos apontam a influência do estresse parental no desenvolvimento e na manutenção de comportamentos externalizantes de crianças (Backer, Heller, \& Henker, 2000; Dessen \& Szelbracikowski, 2004; 2006; Szelbracikowski \& Dessen, 2007).

O estudo de Bolsoni-Silva, Marturano, Pereira e Manfrinato (2006) indica que os problemas de comportamento são preditos por um repertório pobre de habilidades sociais. Também, estudos indicam relações entre autoconceito, habilidades sociais e problemas de comportamento (Leme \& Bolsoni-Silva, 2010).

\section{MÉTODO}

\section{Participantes}

39 crianças com idade entre cinco e sete anos (média de 5,9 anos e desvio padrão de 0,55 ), sendo 22 meninos $(56,4 \%)$ e 17 meninas $(43,6 \%)$, do primeiro ano do ensino fundamental de uma escola privada do interior do Paraná (PR). Participaram 39 mães (média de 32,49 anos e desvio padrão de 4,59) e 25 pais (média de 35,88 anos e desvio padrão de 7,21). As famílias foram categorizadas em quatro tipos: monoparental $(5,1 \%)$, nuclear $(84,6 \%)$, recasada $(2,6 \%)$ e outra $(7,7 \%)$. O número de filhos dessas famílias foi: um filho $(46,2 \%)$, dois filhos $(46,2 \%)$, três filhos $(5,1 \%)$ e quatro filhos $(2,6 \%)$.

\section{Instrumentos}

- Para os Pais:

- Escalas de Qualidade na Interação Familiar EQIF (Weber, Prado, Salvador, \& Brandenburg, 2008), versão para pais. Este instrumento foi proposto para que os pais respondessem sobre suas atitudes e as de seu filho e outros aspectos de sua interação familiar. O instrumento EQIF contém 40 questões, avaliadas pelo sistema de Likert de cinco pontos, e são divididas em nove escalas: quatro escalas sobre práticas parentais: envolvimento, regras e monitoria, comunicação negativa e punição corporal e cinco escalas sobre a percepção dos pais sobre outros aspectos da vida familiar: clima conjugal positivo, clima conjugal negativo, modelo parental, comunicação positiva dos filhos e sentimento dos filhos. Este instrumento produz os seguintes escores: escore total de qualidade na interação familiar, escore de cada prática educativa parental e escore da percepção dos pais sobre outros aspectos da vida familiar, 
- Índice de Estresse Parental - PSI (Abidin, 1995). Composto por 36 itens sobre a percepção que os genitores têm de seu filho e dos sentimentos e reações diante de algum evento ou comportamento do filho. Os itens são avaliados pelo sistema de Likert de cinco pontos e categorizados em três subescalas contendo 12 itens cada, distribuídos da seguinte maneira: sofrimento parental, que mede o sofrimento e a angústia vivenciados pelo genitor em seu papel de pai/mãe, interações disfuncionais entre genitor-criança, que avalia as percepções que os genitores têm de seus filhos que não são compatíveis com suas expectativas, bem como as percepções de suas interações com sua criança que não reforçam o seu papel de pai/mãe, e criança difícil, que focaliza algumas características comportamentais de crianças que as tornam fáceis ou difíceis de serem manejadas. Seus escores são: escore total de estresse parental e escores de cada subescala.

- Escala de Comportamento Social para PréEscolares - PKBS-BR (Dias, Freitas, Del Prette, $\&$ Del Prette, 2011). Produzido originalmente nos EUA (School Social Behavior Scales-2 - Merrel, 2002) e traduzido para o contexto brasileiro, o PKBS-BR é apresentado em uma mesma versão para pais e professores, contendo 34 itens de habilidades sociais e 42 itens de comportamentosproblema. Os itens são avaliados em uma escala tipo Likert de frequência que varia de zero (nunca) a três (frequentemente). No Brasil, o estudo de validação preliminar (Dias et al., 2011) evidenciou propriedades psicométricas satisfatórias quanto à consistência interna da escala total de Habilidades Sociais $(\alpha=0,92)$ e de Comportamentos-problema $(\alpha=0,95)$.

- Para as Crianças:

- Escala de Percepção do Autoconceito Infantil - PAI (Sánchez \& Escribano, 1999). Apresentase aos alunos os 34 desenhos de situações do cotidiano de acordo com o gênero do respondente. Conta-se a história correspondente a cada item e a criança deve marcar, em cada desenho, a figura que mais se parece com ela (esquerda ou direita) e que representa dois pontos de vista diferentes para cada situação. O instrumento analisa o escore total do autoconceito.

\section{Procedimento}

Após a aprovação da pesquisa pelo Comitê de Ética em Pesquisa do Setor de Ciências da Saúde da
UFPR, conforme resolução CNS 196/96 (Registro CEP/SD:675.010.09.02/ CAAE: 0390.0.000.091-09), realizou-se uma apresentação à escola participante sobre os objetivos da pesquisa e, diante da autorização e do consentimento da instituição, os pais foram convidados a participarem de uma reunião sobre a pesquisa. Eles consentiram em participar e autorizaram a participação de seus filhos (Termos de Consentimento Esclarecido e Informado). As coletas de dados com os pais foram realizadas coletivamente na própria escola de forma autoaplicada em uma reunião de pais no início do ano letivo. Após, a autorização dos pais, a coleta de dados com as crianças ocorreu durante dois meses e foi realizada de maneira coletiva com no máximo quatro participantes por grupo nos. Após o término da pesquisa, pais e escola receberam uma devolutiva dos resultados encontrados e sugestões de ações preventivas.

\section{Análise dos dados}

Os dados coletados foram sistematizados utilizandose o software SPSS (Statistical Package for the Social Sciences, versão 15). As análises foram realizadas por meio de medidas descritivas, escores totais e de subescalas e testes estatísticos para correlacionar e relacionar os dados coletados (correlação de Pearson, Qui-quadrado e Regressão Linear). O índice de consistência interna (alfa de Cronbach) foi de $>60 \%$ para a maior parte dos instrumentos.

\section{RESULTADOS E DISCUSSÃO}

Os resultados encontrados nesse estudo foram organizados nas seguintes seções: 1) Relações entre os instrumentos EQIF e PSI, 2) Relações entre os instrumentos EQIF, PSI e PAI, 3) Relações entre os instrumentos EQIF, PSI e PKBS, 4) Modelos de regressão linear entre as variáveis.

\section{Relações entre os instrumentos EQIF e PSI}

Os instrumentos EQIF e PSI tiveram suas consistências internas analisadas por meio do coeficiente alfa de Cronbach, que foi, respectivamente, 0,72 e 0,86 . A relação entre o escore total da EQIF (qualidade na interação familiar) e o escore total de estresse parental revelou que $77 \%$ das crianças que perceberam uma interação familiar de qualidade tinham pais com baixo nível de estresse parental e, ao contrário, $82 \%$ das crianças em uma interação familiar com baixa qualidade tinham pais com alto nível de estresse parental $\left(\chi^{2}=8,22, \mathrm{gl}=1, \mathrm{p}<0,05\right)$. Outros estudos mostram que baixo estresse parental permite interações mais positivas entre genitores-filhos e vice-versa (Dessen \& Szelbracikowski, 2004; Mckelvey et al., 2002). 
A subescala do estresse parental da mãe, interações disfuncionais, teve correlação significativa e inversa com as práticas educativas parentais "envolvimento" $(\mathrm{r}=-0,46, \mathrm{p}<0,01)$, "regras e monitoria" $(\mathrm{r}=-0,40$, $\mathrm{p}<0,05)$ e com aspectos da vida familiar "comunicação positiva" $(\mathrm{r}=-0,33, \mathrm{p}<0,05)$, "modelo parental" $(\mathrm{r}=-0,48, \mathrm{p}<0,01)$ e "sentimento dos filhos pelos pais" $(\mathrm{r}=-0,48, \mathrm{p}<0,01)$. Essa mesma subescala de estresse parental da mãe, interações disfuncionais, teve correlação significativa e direta com a prática educativa parental "comunicação negativa" ( $\mathrm{r}=0,51$, $\mathrm{p}<0,01)$. Os dados sugerem que mães que se sentem satisfeitas em seu papel materno têm melhores práticas educativas parentais e comunicam-se positivamente com seus filhos. Se elas não se sentem satisfeitas em seu papel materno, tendem a xingar, gritar com seus filhos (Dessen \& Szelbracikowski, 2004; Mckelvey et al., 2002).

A subescala do estresse parental da mãe, sofrimento parental, correlacionou-se significativa e inversamente com o aspecto da vida familiar "clima conjugal positivo" $(\mathrm{r}=-0,40, \mathrm{p}<0,05)$ e a subescala, criança difícil, correlacionou-se significativa e diretamente com a prática educativa parental "comunicação negativa" $(\mathrm{r}=0,37, \mathrm{p}<0,05)$ e inversamente com os aspectos da vida familiar "modelo parental" $(\mathrm{r}=-0,71$, $\mathrm{p}<0,01)$ e "sentimento dos filhos" $(\mathrm{r}=-0,33, \mathrm{p}<0,05)$. A análise dos dados permite mencionar que a mãe sentese menos estressada, em seu papel materno, quando se relaciona com seu parceiro de maneira satisfatória. Assim como, a mãe sente-se mais estressada em seu papel materno se a interação com seu filho ocorrer de maneira negativa, ou seja, com xingamentos, raiva e irritabilidade por parte dele.

A percepção do pai sobre o aspecto da vida familiar "sentimentos dos filhos" correlacionou-se significativa e inversamente com todas as subescalas do estresse parental do pai: sofrimento parental $(\mathrm{r}=-0,43, \mathrm{p}<0,05)$, interações disfuncionais $(\mathrm{r}=-0,53, \mathrm{p}<0,01)$ e criança difícil $(r=-0,60, p<0,01)$. A subescala do estresse parental do pai, criança difícil, correlacionou-se significativa e, respectivamente, direta e inversamente com a percepção dos pais sobre os aspectos da vida familiar "clima conjugal negativo" $(r=0,43, p<0,05)$ e "modelo parental" $(\mathrm{r}=-0,46, \mathrm{p}<0,05)$. Pode-se supor que o pai estressa-se quando percebe que seu filho não o ama, não o considera um bom pai e vice-versa. Já quanto mais o pai se estressa com as características comportamentais dos filhos como irritabilidade, mau humor, reações emocionais intensas e não cumprimento de solicitações, mas o pai percebe seu relacionamento conjugal como negativo e não se sente um modelo de pai adequado ao seu filho e vice-versa.
Percebe-se, de maneira geral, que os aspectos da vida familiar identificados pelos pais e mães que mais se correlacionaram com o estresse parental foram sentimentos dos filhos e modelo parental. Em relação apenas a mãe, a comunicação negativa foi uma prática educativa parental correlacionada ao seu estresse parental. Em relação ao pai, a percepção do clima conjugal negativo correlacionou-se com seu estresse parental. Portanto, o estresse parental é um processo complexo e que envolve diversos fatores dos pais, da criança e do contexto familiar (Abidin, 1995; Deater-Deckard, 1998).

\section{Relações entre os instrumentos EQIF, PSI e PAI}

$\mathrm{O}$ instrumento PAI teve sua consistência interna analisada por meio do coeficiente alfa de Cronbach, que foi 0,65 . Em relação ao autoconceito, o resultado encontrado foi uma correlação significativa e direta com o escore total da EQIF (qualidade na interação familiar) $(\mathrm{r}=0,55, \mathrm{p}<0,01)$. Foi encontrada uma correlação significativa e inversa entre autoconceito e uma das escalas de práticas educativas parentais do instrumento EQIF, "comunicação negativa dos pais" ( $\mathrm{r}=-0,45$, $\mathrm{p}<0,05)$. Quanto maior a frequência de comunicação negativa dos pais, ou seja, quanto mais os pais gritam, humilham, ameaçam e xingam seus filhos, mais baixo é o autoconceito dos mesmos. Este fato ocorre porque é o ambiente social afetivo e imediato (a família) que fornece informações sobre quem são as crianças, o que as tornará capazes (ou não) de discriminar, descrever e controlar seus próprios comportamentos, com o passar dos anos (Loos \& Cassemiro, 2010). Outra correlação significativa e inversa encontrada foi entre o autoconceito e a escala de prática educativa parental da mãe chamada de "punição corporal" $(\mathrm{r}=-0,47, \mathrm{p}<0,05)$. A punição corporal traz à criança uma percepção enfraquecida de si mesma, que compromete suas possibilidades de sucesso e uma relação coercitiva, pois ela passa a entender que quem ama tem o direito de machucar, podendo trazer dificuldades funcionais em seus relacionamentos futuros (Weber, 2008).

Esses resultados sustentam a importância da qualidade das interações familiares na construção do autoconceito das crianças, enfatizando o impacto da prática educativa parental, comunicação negativa, como fator de risco para a formação de um autoconceito empobrecido (Bolsoni-Silva \& Marturano, 2002; Loos \& Cassemiro, 2010; Rocha et al., 2011; Weber et al., 2004).

\section{Relações entre os instrumentos EQIF, PSI e PKBS}

$\mathrm{O}$ instrumento PKBS teve sua consistência interna analisada por meio do coeficiente alfa de Cronbach, 
que foi de 0,90 . Um dos resultados analisados revelou relação significativa e direta entre o escore total das habilidades sociais das crianças e o escore total da qualidade na interação familiar $\left(\chi^{2}=8,71, \mathrm{gl}=1\right.$, $\mathrm{p}<0,05)$. Quando o relacionamento dos pais com os filhos é sustentado por fatores positivos - como regras claras, informações sobre as contingências em vigor para os comportamentos sociais - e a criança percebe o monitoramento a que está sujeita, existe maior probabilidade do desenvolvimento de relações saudáveis em todos os seus contextos de interações sociais (Del Prette \& Del Prette, 2006). O escore total da EQIF (qualidade na interação familiar) correlacionou-se significativa e negativamente com o escore dos problemas de comportamento externalizantes das crianças $(\mathrm{r}=-0,42, \mathrm{p}<0,05)$. Ou seja, o ambiente familiar pode premiar ou modelar os comportamentos inadequados, nesse caso, os externalizantes, e ignorar os adequados ou adotar medidas pouco efetivas para promovê-los (Del Prette \& Del Prette, 2006).

Foram encontradas correlações significativas e diretas entre o escore total das habilidades sociais das crianças e a escala de prática educativa parental "envolvimento parental" $(\mathrm{r}=0,50, \mathrm{p}<0,01)$ e as escalas de percepção dos pais sobre os aspectos da vida familiar "modelo parental" $(\mathrm{r}=0,48, \mathrm{p}<0,05)$ e "sentimento das crianças pelos seus pais" $(r=0,64, p 0,01)$. As habilidades sociais dos filhos são maiores quando os pais envolvem-se na vida deles (dizendo o quanto ele é importante, demonstrando carinho etc.), quanto maior o modelo parental (fazem o que dizem etc.) e quanto maior é o sentimento dos filhos (me sinto amado pelo meu pai etc.). Certamente, pais mais envolvidos estão mais disponíveis a ajudarem a criança a discriminar padrões de interações funcionais, bem como servem de modelos a essas interações e a valores de conduta (Barros \& Del Prette, 2007). Isso se manifesta nos filhos por meio de um sentimento mais positivo e, consequentemente, interações sociais mais prósperas.

$\mathrm{O}$ escore dos problemas de comportamento externalizantes das crianças correlacionou-se com a escala de percepção dos pais sobre o aspecto da vida familiar "sentimento das crianças pelos seus pais" $(\mathrm{r}=-0,56, \mathrm{p}<0,01)$. Pode-se supor que, quanto menos satisfeitos os filhos estão com seus pais, na percepção desses, mais os filhos utilizam estratégias agressivas e opositivas em suas interações para atingirem seus objetivos.

Em relação ao escore do estresse parental, o resultado encontrado foi entre o escore dos problemas de comportamentos externalizantes das crianças $(\mathrm{r}=0,41, \mathrm{p}<0,05)$. Pode-se inferir, a partir desse dado, que pais que se estressam com a educação dos filhos, ou seja, os percebem de maneira negativa, podem agir de forma negativa com os mesmos, propiciando às crianças a emissão de comportamentos externalizantes (Dessen \& Szelbracikowski, 2004; 2006; Szelbracikowski \& Dessen, 2007). Além disso é necessário enfatizar outra inferência: crianças com comportamentos externalizantes tendem a dificultar a interação genitor-criança e, desta forma, aumentam as chances de estresse parental.

\section{Modelos de regressão linear entre as variáveis}

Buscando aprofundar a compreensão das relações explicativas entre as variáveis da presente pesquisa, utilizou-se o método da regressão linear múltipla, que consiste em avaliar o efeito de variáveis independentes (VIs) sobre uma variável dependente (VD). Foram selecionadas, para esta análise, duas variáveis independentes para cada variável dependente testada, de acordo com o critério de definição de 15 casos por variável independente.

Modelo de regressão linear múltipla 1: O modelo formado pelas variáveis "punição corporal" utilizado pelos pais e habilidades sociais dos filhos explicaram $49 \%$ da variação dos problemas de comportamento (externalizantes e internalizantes) das crianças. A variável punição corporal $(\beta=0,46, p<0,01)$ apresentou maior contribuição explicativa, enquanto a variável, habilidades sociais $(\beta=-0,41, \mathrm{p}<0,05)$, apresentou uma contribuição ligeiramente menor.

A literatura aponta para a punição corporal como uma das práticas educativas de grande influência negativa ao desenvolvimento infantil (Brandenburg \& Weber, 2005; Oliveira \& Caldana, 2009). Os pais que utilizam a punição corporal, tendem a não ser contingentes no uso de reforçamento positivo para comportamentos pró-sociais dos filhos, pois esses comportamentos podem ser frequentemente ignorados ou respondidos de forma inapropriada, não sendo reforçados ou até mesmo punidos pelos pais. Consequentemente, essa prática educativa parental leva ao fortalecimento de comportamentos coercitivos, os quais são utilizados pelas crianças para sobreviverem no ambiente social aversivo. Pesquisas anteriores também apontam as punições corporais utilizadas pelos pais como preditoras de problemas de comportamento das crianças (Ferreira \& Marturano, 2002).

As habilidades sociais (HS) recebem cada vez mais atenção de psicólogos, psiquiatras e educadores por se constituírem um indicador bastante importante de ajustamento psicossocial e de perspectivas positivas para o desenvolvimento (Del Prette \& Del Prette, 
2006). As dificuldades interpessoais que caracterizam os problemas de comportamento decorrem basicamente de um repertório pobre de HS, como por exemplo, empatia, expressão de sentimentos e resolução de problemas (Del Prette \& Del Prette, 2006). Certamente, um repertório pobre de HS dificulta às crianças interagir de forma positiva com colegas, professores e familiares, e, desta forma, é um preditor de problemas de comportamento (Bolsoni-Silva et al., 2006; Bolsoni-Silva \& Del Prette, 2003; Del Prette \& Del Prette, 2002).

Modelo de regressão linear múltipla 2: $\mathrm{O}$ modelo formado pelas variáveis habilidades sociais e autoconceito das crianças explicaram $47 \%$ da variação dos problemas de comportamento externalizantes. Observa-se que a variável, habilidades sociais $(\beta=-0,55, \quad p<0,01)$, apresenta maior contribuição explicativa ao modelo e a variável autoconceito ( $\beta=-0,39, p<0,001)$ uma contribuição sensivelmente menor.

Este segundo modelo de regressão linear múltipla aponta para uma direção que verifica a preocupação geral de pais e educadores: as crianças com problemas de comportamento externalizantes. Esse resultado é corroborado pela literatura que indica relações entre habilidades sociais, autoconceito e problemas de comportamento (Del Prette \& Del Prette, 2006; Leme \& Bolsoni-Silva, 2010). O ambiente familiar e essencial na promoção do autoconceito (Rocha et al., 2011) e na construção do repertorio de habilidades sociais dos filhos e são eles, autoconceito e habilidades sociais, que previnem dificuldades interpessoais e outros transtornos psicológicos na infância (Del Prette \& Del Prette, 2006).

Modelo de regressão linear múltipla 3: O modelo formado pelas variáveis estresse parental e habilidades sociais dos filhos explica $44 \%$ da variação da qualidade na interação familiar. Observa-se, no modelo de regressão 3 , que a variável estresse parental $(\beta=-0,46$, $\mathrm{p}<0,05)$ apresenta maior contribuição explicativa ao modelo, enquanto a variável habilidades sociais $(\beta=0,38, p<0,05)$ oferece uma contribuição menor. $\mathrm{O}$ estresse parental relacionado às escalas de práticas educativas parentais, que medem parte da qualidade na interação familiar, já foi apontado pela literatura (Mckelvey et al., 2002), ou seja, a irritabilidade e o sofrimento parental diante de seus papéis contribuem para que os pais tenham interações com seus filhos que seriam mais aversivas ou negativas. O repertório de HS dos filhos também influencia diretamente a qualidade na interação familiar, ou seja, quanto mais adequado o repertório de HS dos filhos mais os pais utilizam práticas educativas positivas como, por exemplo, a monitoria, o afeto, o envolvimento, o modelo etc.

\section{CONSIDERAÇÕES FINAIS}

O presente estudo trouxe contribuições fundamentais ao tema família e desenvolvimento infantil. Os resultados mostraram que os pais sabem se comportar de maneira adequada em relação às diversas situações vivenciadas em seu papel parental e percebem os aspectos da vida familiar, relacionamento conjugal e comunicação, como satisfatórios. Esses aspectos os protegem de se perceberem como estressados e seus papéis parentais. A interação familiar de baixa qualidade ("comunicação negativa" e "punição corporal") compromete a construção do autoconceito positivo e de um bom repertório de habilidades sociais das crianças. Tanto a interação familiar de baixa qualidade quanto o estresse parental relacionaram-se com problemas de comportamento externalizantes das crianças. De maneira geral, espera-se que os resultados encontrados nesse estudo estimulem ainda mais pesquisas futuras a identificar as variáveis que contribuem aos desenvolvimentos familiar e infantil saudáveis.

\section{REFERÊNCIAS}

Abidin, R. R. (1995). Parenting stress index. Odessa: Psychological Assessement Resources.

Alvarenga, P. \& Piccinini, C. A. (2001). Práticas educativas maternas e problemas de comportamento em pré-escolares. Psicologia: Reflexão e Crítica, 14(3), 449-459.

Backer, B. L., Heller, T. L., \& Henker, B. (2000). Expressed emotion, parenting stress and adjustment in mothers of young children with behavior problems. Journal of Child Psychology and Psychiatry, 41(7), 907-915.

Barros, S. K. S. N. \& Del Prette, A. (2007). Um treinamento de habilidades sociais para pais pode beneficiar os filhos na escola? Revista da SPTM, 11(1), 107-123.

Bayer, J. K., Sanson, A. V., \& Hemphill, S. A. (2006). Parental influences on early childhood internalizing difficulties. Journal of Applied Developmental Psychology, 27, 542-559.

Bolsoni-Silva, A. T. \& Del Prette, A. (2003). Problemas de comportamento: um panorama da área. Revista Brasileira de Terapia Comportamental e Cognitiva, 5(2), 91-103.

Bolsoni-Silva, A. T. \& Loureiro, S. R. (2011). Práticas educativas parentais e repertório comportamental infantil: comparando crianças diferenciadas pelo comportamento. Paidéia, 21(48), 61-71.

Bolsoni-Silva, A. T. \& Marturano, E. M. (2002). Práticas educativas e problemas de comportamento: uma análise à luz das habilidades sociais. Estudos de Psicologia (Natal), 7(2), 227-235.

Bolsoni-Silva, A. T. \& Marturano, E. M. (2008). Habilidades sociais educativas parentais e problemas de comportamento: comparando pais e mães de pré-escolares. Aletheia, 27, 126-138. 
Bolsoni-Silva, A. T. \& Marturano, E. M. (2010). Relacionamento conjugal, problemas de comportamento e habilidades sociais de pré-escolares. Psicologia: Teoria e Pesquisa, 26(1), 67-75.

Bolsoni-Silva, A. T., Marturano, E. M., Pereira, V. A., \& Manfrinato, J. W. S. (2006). Habilidades sociais e problemas de comportamento de pré-escolares: comparando avaliações de mães e de professoras. Psicologia: Reflexão e Crítica, 19(3), 460-469.

Bolsoni-Silva, A. T., Silveira, F. F., \& Marturano, E. M. (2008). Promovendo habilidades sociais educativas parentais na prevenção de problemas de comportamento. Revista Brasileira de Terapia Comportamental e Cognitiva, 10(2), 125-142.

Brandenburg, O. J. \& Weber, L. N. D. (2005). Revisão de literatura da punição corporal. Interação em Psicologia, 9(1), 91-102.

Carmo, P. H. B. \& Alvarenga, P. (2012). Praticas educativas coercitivas de mães de diferentes níveis socioeconômicos. Estudos de Psicologia, 17(2), 191-197.

Cia, F., Pamplin, R. C. O., \& Del Prette, Z.A. P. (2006). Comunicação e participação pais-filhos: correlação com habilidades sociais e problemas de comportamento dos filhos. Paidéia, 6(35), 395-406.

Deater-Deckard, K. (1998). Parenting stress and child adjustment: some old hypotheses and new questions. Clinical Psychology: Science and Practice, 5(3), 314-332.

Del Prette, A. \& Del Prette, Z. A. P. (2002). Psicologia das relações interpessoais: vivências para o trabalho em grupo ( $2^{\mathrm{a}}$ ed.). Rio de Janeiro: Vozes.

Del Prette, Z. A. P. \& Del Prette, A. (2006). Psicologia das habilidades sociais na infância: teoria e prática (2a ed.). Rio de Janeiro: Vozes.

Del Prette, Z. A. P. \& Del Prette, A. (2008). Um sistema de categorias de habilidades sociais educativas. Paidéia, 18(41), 517-530.

Dessen, M. A. \& Polonia, A. C. (2007). A família e a escola como contextos de desenvolvimento humano. Paidéia, 36, 21-32.

Dessen, M. A. \& Szelbracikowski, A. C. (2004). Crianças com problemas de comportamento exteriorizado e a dinâmica familiar. Interação em Psicologia, 8(2), 171-180.

Dessen, M. A. \& Szelbracikowski, A. C. (2006). Estabilidades e mudanças em padrões familiares de crianças com problemas de comportamento exteriorizado. Paidéia, 16(33), 71-80.

Dias, T. P., Freitas, L. C., Del Prette, Z. A. P., \& Del Prette, A. (2011). Validação da escala de comportamentos sociais de pré-escolares para o Brasil. Psicologia em Estudo, 16, 447-457.

Ferreira, M. C. T. \& Marturano, E. M. (2002). Ambiente familiar e os problemas de comportamento apresentados por crianças com baixo desempenho escolar. Psicologia: Reflexão e Crítica, $15,35-44$

Freitas, A. P. C. O. \& Piccinini, C. A. (2010). Práticas educativas parentais em relação ao filho único e ao primogênito. Estudos de Psicologia, Campinas, 27(4), 515-528.

Kreppner, K. (2000). The child and the family: interdependence in developmental pathways. Psicologia: Teoria e Pesquisa, 16(1), 11-22.

Leme, V. B. R. \& Bolsoni-Silva, A. T. (2010). Habilidades sociais educativas parentais e comportamentos de pré-escolares. Estudos de Psicologia, 15(2), 161-173.

Lengua, L. J. \& Kovacs, E. A. (2005). Bidirectional associations between temperament and parenting and the prediction of adjustment problems in middle childhood. Apllied Developmental Psychology, 26, 21-38.

Loss, H. \& Cassemiro, L. F. K. (2010). Percepção sobre a qualidade da interação familiar e crenças autorreferenciadas em crianças. Estudos de Psicologia, Campinas, 27(3), 293-303.

Macarini, S. M., Martins, G. D. F., Minetto, M. F., \& Vieira, M. L. (2010). Práticas parentais: uma revisão da literatura brasileira. Arquivo Brasileiro de Psicologia, 62(1), 119-134.
Marin, A. H., Piccinini, C. A., \& Tudge, J. R. H. (2011). Praticas educativas maternas e paternas aos 24 e aos 72 meses de vida da criança. Psicologia: Teoria e Pesquisa, 27(4), 419-427.

Merrell, K. W. (2002). Preschool and kindergarten behavior scales (2nd ed.) Austin, Tx: Pro-Ed.

Mckelvey, L. M., Fitzgerald, H. E., Schiffman, R. F., \& Von Eye, A. (2002). Family stress and parent-infant interaction: the mediating role of coping. Infant Mental Health Journal, 23(2)164-181.

Oliveira, T. T. S. \& Caldana, R. H. L. (2009). Educar e punir? Concepções e práticas educativas de pais agressores. Estudos e Pesquisas em Psicologia, 9(3), 679-694.

Oliveira, E. O., Marin, O. H., Pires, F. B., Frizzo, G. B., Ravanello, T., \& Rossato, C. (2002). Estilos parentais autoritário e democrático-recíproco intergeracionais, conflito conjugal e comportamentos de externalização e internalização. Psicologia: Reflexão e Crítica, 1(1), 1-11.

Pettit, G. S. \& Bates, J. E. (1989). Family interaction patterns and children's behavior problems from infancy to 4 years. Developmental Psychology, 25(3), 413-420.

Pereira-Silva, N. L. \& Dessen, M. A. (2006). Famílias de crianças com síndrome de down: sentimentos, modos de vida e estresse parental. Interação em Psicologia, 10(2), 183-194.

Rocha, G. V. M., Ingberman, Y. K., \& Breus, B. (2011). Analise da relação entre praticas parentais e o autoconceito de préescolares. Revista Brasileira de Terapia Comportamental e Cognitiva, 13(1), 87-106.

Sampaio, I. T. A. \& Vieira, M. L. (2010). A influência do gênero e ordem de nascimento sobre as práticas educativas parentais. Psicologia: Reflexão e Crítica, 23(2), 198-207.

Sánchez, A. V. \& Escribano, E. A. (1999). Medição do Autoconceito. Bauru: Edusc.

Szelbracikowski, A. C. \& Dessen, M. A. (2007). Problemas de comportamentos exteriorizado e as relações familiares: revisão de literatura. Psicologia em Estudo, Maringá, 12(1), 33-40.

Wagner, A., Ribeiro, L. S., Arteche, A. X., \& Bornholdt, E. A. (1999). Configuração familiar e o bem-estar psicológico dos adolescentes. Psicologia: Reflexão e Crítica, 12(1), 147-156.

Weber, L. N. D. \& Moura, V. F. (2008). Interações familiares e comportamento anti-social. In L. N. D. Weber (Org.). Família e desenvolvimento: visões interdisciplinares (pp. 102-116). Curitiba: Juruá.

Weber, L. N. D. (2008). Interações entre família e desenvolvimento. In L. N. D. Weber (Org.), Família e desenvolvimento: visões interdisciplinares. (pp. 9-20). Curitiba: Juruá.

Weber, L. N. D., Prado, P. M., Salvador, A. P. V., \& Brandenburg, O. J. (2008). Construção e confiabilidade das escalas de qualidade na interação familiar. Psicologia Argumento, 26(52), 55-65.

Weber, L. N. D., Viezzer, A. P., \& Brandenburg, O. J. (2004). O uso de palmadas e surras como prática educativa. Estudos de Psicologia, 9, 227-237.

\section{Autores:}

Gisele Regina Stasiak - Professora do Curso de Psicologia da Faculdade Sant'Ana e Mestre em Educação pela UFPR. Doutoranda em Educação pela UFPR. Lidia Natalia Dobrianskyj Weber - Professora do Departamento de Psicologia e do Mestrado em Educação da UFPR, Doutora em Psicologia Experimental pela USP e Pós-doutora em Processos de Desenvolvimento Humano e Saúde pela UnB.

Claudia Tucunduva - Mestre em Educação pela UFPR.

\section{Endereço para correspondência:}

Gisele Regina Stasiak

Rua Joaquim de Paula Xavier, 561

CEP 84050-000 Ponta Grossa, PR, Brasil

E-mail: giselestasiak@yahoo.com.br

Recebido em: 13.11 .13

Aceito em: 10.07.14 